\title{
L'évaluation à distance vécue par les étudiants en situation de handicap
}

\section{Distance assessment experienced by students with disabilities}

\section{La evaluación a distancia experimentada por estudiantes con discapacidad}

https://doi.org/10.52358/mm.vi9.238

Valérie Wathelet, conseillère pédagogique

Université de Namur, Belgique

valerie.wathelet@unamur.be

Sandrine Vieillevoye, conseillère pour les étudiants en situation de handicap

Université de Namur, Belgique

sandrine.vieillevoye@unamur.be

\section{RÉSUMÉ}

Dans le cadre de la pandémie, depuis la session de juin 2020, de nombreux examens habituellement prévus en présentiel ont été menés à distance. À l'université, en avril 2020, les services d'accompagnement des étudiants en situation de handicap et les cellules technopédagogique et pédagogique ont uni leurs forces pour soutenir les enseignants dans l'aménagement de leurs examens et informer les étudiants pour s'y préparer et les passer. En février 2021, une enquête en ligne a été menée auprès des étudiants en situation de handicap; nous avons pu mettre en évidence des difficultés rencontrées, ce qui les aide et ce qui pourrait les aider. Cela a permis de dégager quelques bonnes pratiques pour les enseignants et pour les étudiants.

Mots-clés : évaluations, distanciel, étudiants en situation de handicap, enquête 
In the context of the pandemic, since the June 2020 session, many exams usually scheduled face-to-face have been conducted at a distance. At the institutional level, in April 2020, the support service for students with disabilities and the technical and pedagogical units joined forces to support teachers in organizing their exams and informing students how to prepare and take them. In February 2021, students with disabilities received an online surveyThis survey allowed us to identify difficulties encountered by students, what helps them and what could help them. This allowed us to identify some good practices for teachers and students.

Keywords: assessments, distance learning, students with disabilities, survey

RESUMEN

En el contexto de la pandemia, desde la sesión de junio de 2020, muchos exámenes generalmente programados en persona se han realizado de forma remota. A nivel institucional, en abril de 2020, los servicios de apoyo a los estudiantes con discapacidades y las unidades tecnopedagógicas y pedagógicas unieron fuerzas para apoyar a los profesores en la organización de sus exámenes e informar a los estudiantes para prepararlos y presentarlos. En febrero de 2021, se realizó una encuesta en línea entre estudiantes con discapacidades, pudimos identificar dificultades encontradas, y lo que les ayuda y lo que podría ayudarles. Esto permitió identificar algunas buenas prácticas para profesores y estudiantes.

Palabras clave: evaluaciones, educación a distancia, estudiantes con discapacidades, encuesta

\section{Introduction}

À la suite de la pandémie, l'Université de Namur a fait le choix de mener les trois sessions d'examens d'avril 2020 à janvier 2021 principalement à distance.

Pour l'adaptation de ces sessions au distanciel, une première réflexion pédagogique a été menée sur les modalités à mettre en place pour respecter l'alignement pédagogique (Biggs, 1996) entre les activités de cours (qui ont été réalisées à distance) et les évaluations. En effet, les objectifs pédagogiques initialement prévus n'ont pas tous pu être maintenus tels quels. Certains savoirs, savoir-faire et savoir-être ont été transposés. Par exemple, les activités de travaux pratiques en laboratoire n'ont pas pu être réalisées et ont été adaptées en présentant la manipulation sous forme d'une vidéo et en donnant des résultats à analyser. Les enseignants ont pu bénéficier d'une aide lors de l'adaptation des modalités de leurs évaluations au distanciel. Les enjeux de cette réflexion (Audet, 2011) étaient de s'assurer que l'évaluation mesure effectivement la maitrise des connaissances et des compétences 1) nécessaires à la réussite et 2) en tenant compte des adaptations mises en place dans la phase d'enseignement à distance. Cela participe à la qualité de l'évaluation :

a) sur le plan pédagogique : alignement pédagogique entre les objectifs, les activités menées au cours et ce qui est évalué ainsi que le soin apporté à la rédaction des questions d'examen (l'interaction avec l'enseignant étant limitée); 
b) sur le plan organisationnel : l'outil utilisé doit être maitrisé par l'étudiant et les modalités ainsi que les consignes pour accéder et présenter l'examen doivent être complètes et précises afin de limiter l'impact du numérique sur la mesure elle-même.

À la suite des nouvelles modalités dégagées dans le cadre de l'évaluation à distance, la réflexion pédagogique a été poursuivie pour déterminer les aménagements raisonnables prévus dans le plan d'accompagnement individualisé des étudiants en situation de handicap (ESH). La mise en place des dispositifs d'évaluation à distance est brièvement décrite dans la partie suivante.

Pour donner suite à l'expérience de trois sessions, nous avons mené une enquête auprès des ESH pour recueillir leur vécu lors des examens à distance. La méthodologie ainsi que les résultats et leur analyse sont présentés dans les parties 3 à 5 . Une conclusion sous forme de bonnes pratiques et des recommandations clôturent cet article.

\section{Mise en place des dispositifs d'évaluation à distance}

Pour transposer les évaluations en ligne pour tous les étudiants avec une attention particulière aux ESH, la cellule en charge des technologies de l'information et de la communication de l'enseignement (TICE), l'équipe pédagogique (PUNCH - Pédagogie Universitaire Namuroise en CHangement) et le Service d'accompagnement des ESH ont uni leurs forces.

\section{Le passage du présentiel au distanciel}

Organiser une session à distance nécessite une maitrise des outils technologiques utilisés tant par les enseignants-examinateurs que par les étudiants. L'équipe technopédagogique a choisi de se baser prioritairement sur les outils utilisés habituellement au sein de l'Université, car cela suppose une meilleure maitrise de la part des utilisateurs et un soutien assuré de l'Université. II s'agit d'une plateforme LMS (Learning Open Source) de type Moodle et d'un logiciel de visioconférence, Teams. Pour vérifier la maitrise des outils utilisés et l'accès à du matériel approprié par les étudiants, une simulation d'examen leur a été proposée un mois avant la session d'examens. Elle leur donnait accès à un passeport leur permettant de réaliser leurs examens. Cela a également permis de relever les soucis matériels rencontrés par certains et de leur proposer des solutions (prêt ou présentation d'examens sur le site, etc.).

Tout d'abord, pour les types d'évaluation majoritaires (tests, travaux écrits, démonstration de compétence) (Liang et Creasy, 2004; Nizet et al., 2016), plusieurs scénarios d'examens ont été proposés et un organigramme Figure 1) a été créé pour aider les enseignants dans leurs choix d'adaptation. Ensuite des tutoriels, des séances d'information et des consultations individuelles leur ont été proposés. Les méthodes d'évaluation les plus courantes dans le contexte d'apprentissage en ligne étaient basées sur les tests, les devoirs de Moodle ainsi que l'utilisation de Teams pour garder un contact avec les étudiants. Une vérification des examens essentiellement numérique a été effectuée par un technopédagogue pour tous les enseignants qui en faisaient la demande et un retour leur a été donné. Les sollicitations sur l'aspect pédagogique avaient généralement eu lieu préalablement à l'encodage de l'examen. 


\section{Figure 1}

Organigramme permettant le choix d'un dispositif d'examen à distance

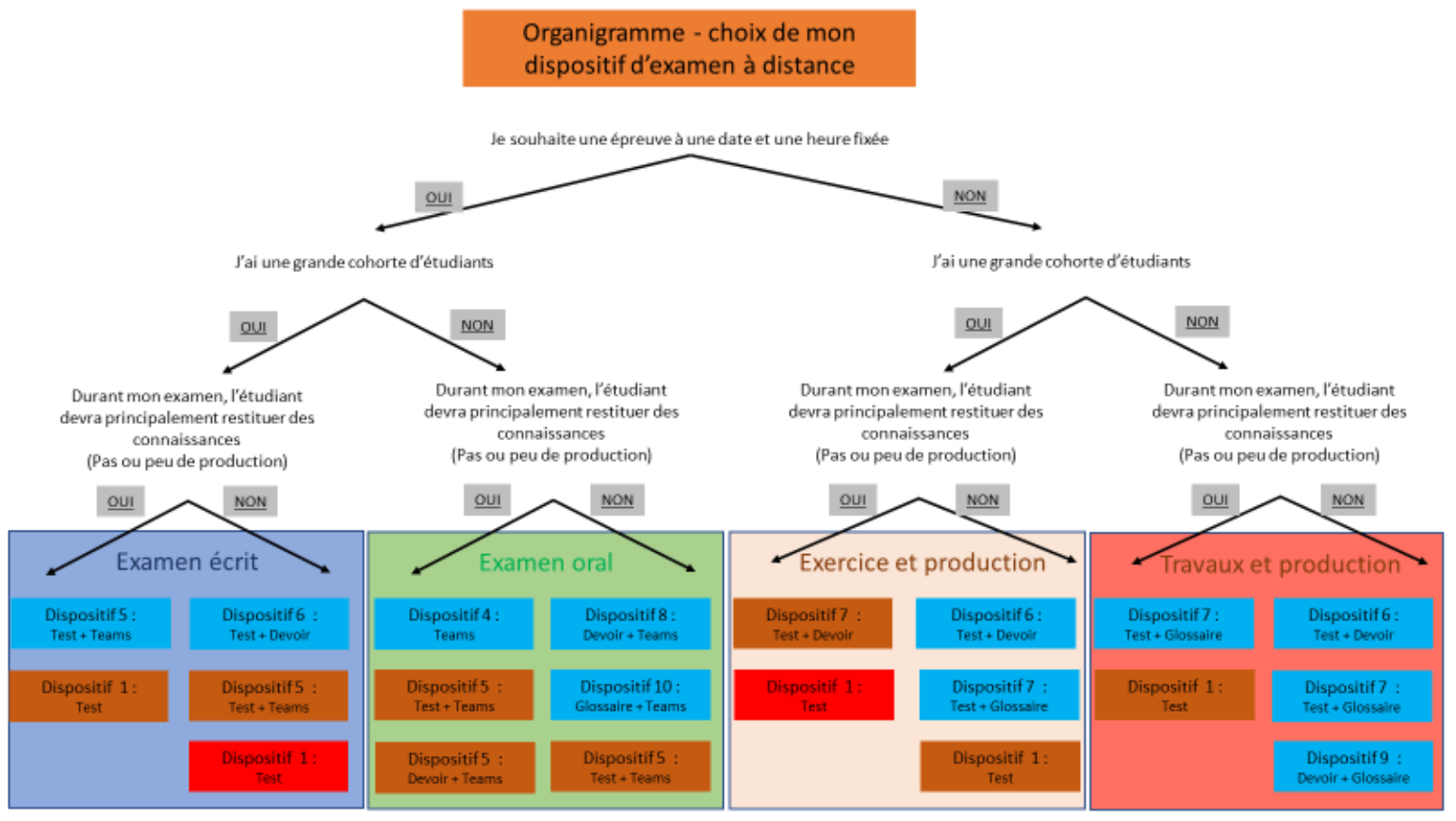

\section{Dans le cadre de l'enseignement inclusif}

La réflexion sur la mise à distance des examens pour les ESH a tout d'abord consisté à rappeler ou à expliquer l'origine de chaque aménagement et à justifier son maintien. Il était évident de veiller à maintenir la qualité des aménagements proposés. Ils constituent en effet pour les ESH « le seul moyen de montrer leurs réelles capacités » (Keunen, 2020). Ensuite, les technopédagogues ont proposé des aménagements en fonction de leur origine. Un exemple est donné pour un aménagement dans le tableau 1. Enfin, ils ont rédigé des tutoriels expliquant le paramétrage nécessaire pour certains aménagements et des réponses aux questions fréquemment posées. Toutes ces informations ont été mises à la disposition des enseignants, des étudiants, des conseillers pédagogiques facultaires et des personnes relais. 


\section{Tableau 1}

Exemple d'aménagement

\begin{tabular}{|c|c|}
\hline Aménagement & $\begin{array}{l}\text { Lors des évaluations par questions à choix multiples (QCM), avoir } \\
\text { la possibilité de noter un commentaire à côté de la case cochée. }\end{array}$ \\
\hline Origine & $\begin{array}{l}\text { Cet aménagement peut répondre aux difficultés de compréhension } \\
\text { des questions selon le sens commun malgré une bonne maitrise de } \\
\text { la matière (dans le cas d'un trouble du spectre autistique ou de } \\
\text { capacités intellectuelles de haut niveau). }\end{array}$ \\
\hline $\begin{array}{l}\text { Justification du } \\
\text { maintien }\end{array}$ & $\begin{array}{l}\text { Avec la distance, les étudiants ont moins l'occasion de poser des } \\
\text { questions de clarification aux enseignants. Il est donc important de } \\
\text { maintenir la possibilité de noter un commentaire en lien avec la } \\
\text { case cochée. }\end{array}$ \\
\hline \multirow[t]{3}{*}{$\begin{array}{l}\text { Proposition pour la } \\
\text { version en ligne }\end{array}$} & $\begin{array}{l}\text { Permettre la remise des feuilles de commentaires après le temps } \\
\text { de l'examen soit par mail à l'enseignant soit dans un espace de } \\
\text { type « Devoir ». }\end{array}$ \\
\hline & $\begin{array}{l}\text { Il est donc nécessaire de prévoir un temps pour numériser ces } \\
\text { feuilles (ou les prendre en photo) et les transmettre. }\end{array}$ \\
\hline & $\begin{array}{l}\text { Si les autres étudiants ne doivent pas déposer leurs feuilles, } \\
\text { restreindre ce devoir aux étudiants concernés (par individu, ou } \\
\text { créer un groupe s'ils sont plusieurs). }\end{array}$ \\
\hline
\end{tabular}

\section{Méthodologie de l'étude}

L'étude sur le vécu des évaluations à distance pour les ESH a été menée sur la base d'une enquête constituée de 20 questions réparties en 4 parties traitant 1) des caractéristiques (individuelles et matérielles) des étudiants lors de la session de janvier 2021, 2) des aménagements dont ils disposaient, 3 ) de leur ressenti pour les examens en ligne et enfin 4) de l'encadrement de la part du service pour les ESH.

L'enquête a été menée via un questionnaire en ligne utilisant l'outil LimeSurvey. Tous les ESH de l'UNamur ont été contactés par courriel en février 2021. Ils ont disposé d'un mois pour y répondre. Un rappel a été envoyé une semaine plus tard. Leurs réponses étaient anonymes. 


\section{Présentation des résultats}

Parmi les 125 étudiants contactés par courriel, 98 ont répondu au questionnaire, dont 13 uniquement aux questions obligatoires. Ces 125 étudiants (sur 6765 étudiants inscrits à l'Université de Namur) bénéficiaient du statut d'ESH lors de la session d'évaluation du mois de janvier. Les ESH rencontrent des difficultés liées à un handicap ou une déficience avérée, une maladie invalidante, un trouble cognitif. Les troubles « dys » (environ $25 \%$ des ESH) et les troubles de l'attention (environ $33 \% \mathrm{ESH}$ ) sont les difficultés les plus fréquemment rencontrées au sein des ESH inscrits à l'Université de Namur. Une large majorité des étudiants (90) sont inscrits en bachelier, 5 en master et 3 suivent l'agrégation de l'enseignement secondaire supérieur (AESS) (Figure 2a). La répartition des étudiants dans les différentes facultés est présentée dans la Figure $2 \mathrm{~b}$. Cela représente $0,7 \%$ en Faculté d'informatique et $2,1 \%$ en Faculté de philosophie et lettres.

Figure 2

Répartition des étudiants (en nombre d'individus) en situation de handicap en fonction de : a) l'année d'étude de l'inscription principale et b) la faculté

(a)

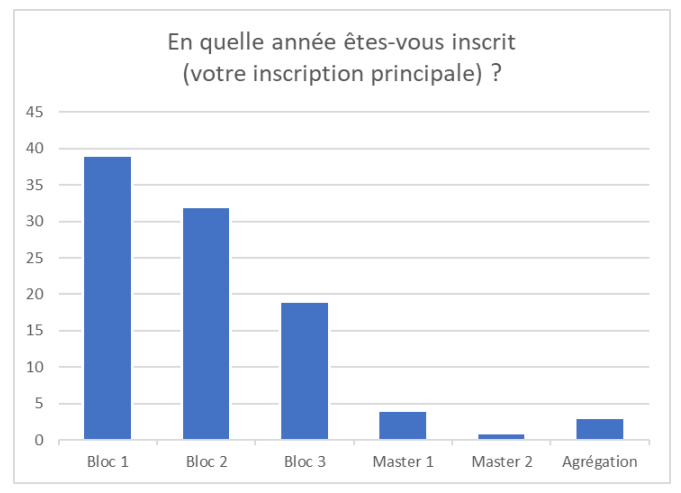

(b)

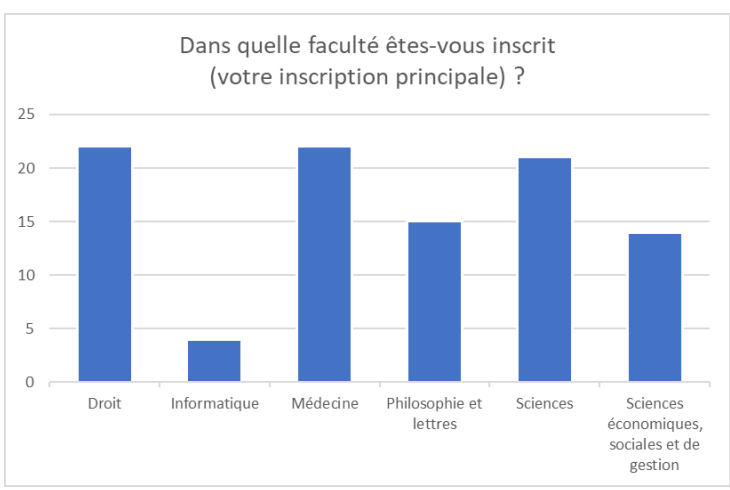

Sur le plan de la qualité du matériel informatique dont les ESH disposent pour leurs examens, 91 en sont satisfaits, voire très satisfaits. La qualité de l'environnement de travail et de la connexion Internet leur pose plus de soucis (figure 3 ). Plus d'un quart sont de « insatisfaits » à « plus ou moins satisfaits ». 


\section{Figure 3}

Répartition des étudiants (en nombre d'individus) en situation de handicap en fonction de leur satisfaction de la qualité de : a) leur environnement de travail b) leur connexion Internet

(a)

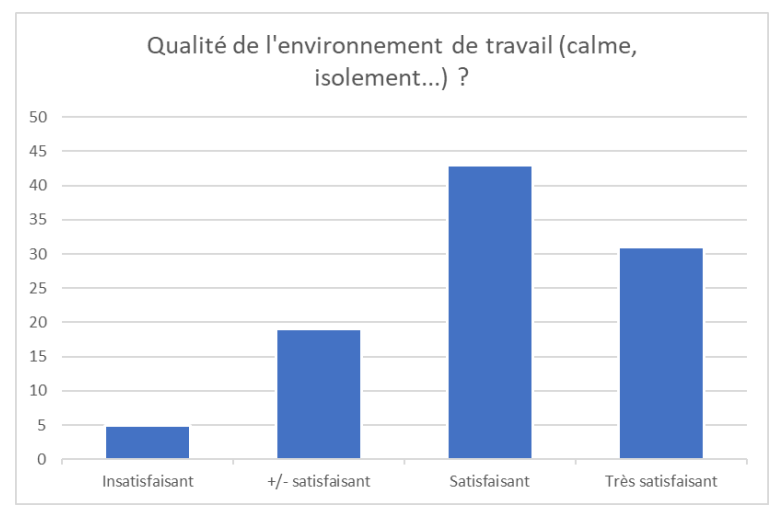

(b)

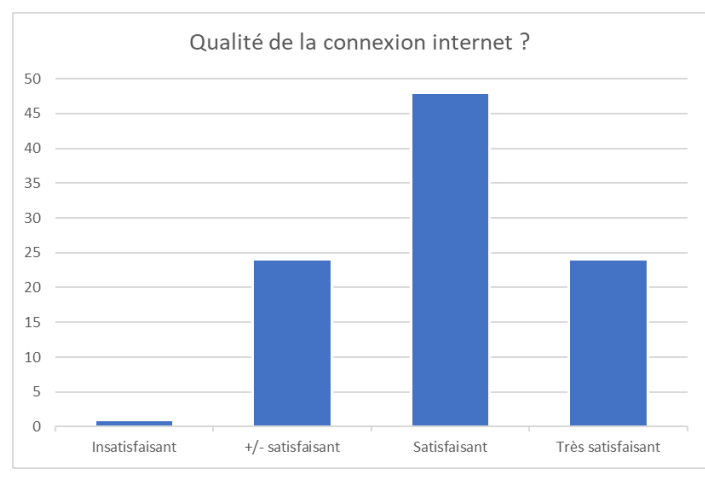

Les étudiants passent en moyenne 6 examens au total, dont 3 en ligne. Certains examens sont demeurés en présentiel. La médiane des examens en ligne se situe à 2 et à 6 pour ceux en présentiel. Cela signifie que la moitié des étudiants ont réalisé au maximum 2 examens en ligne. II s'agit principalement de tests chronométrés reprenant des questions ouvertes et/ou des questions fermées à correction automatisée (de type QCM, glisser-déposer, etc.).

Nous vous proposons ci-dessous les réponses aux trois questions ouvertes qui nous ont semblé les plus riches. La première de ces questions était la suivante : « En lien avec votre situation de handicap, qu'estce qui vous a mis en difficulté pour les examens en ligne? ».

Comme indiqué dans le tableau 2, 29 étudiants rapportent avoir été mis en difficulté par rapport à la question du temps. Ils expriment avoir eu l'impression que, pour éviter la tricherie, la durée de l'examen avait été réduite. Plusieurs d'entre eux ont manqué de temps malgré le tiers-temps supplémentaire octroyé.

Le format du questionnaire a mis 21 étudiants en difficulté. Ceux-ci rapportent que la lecture des questions est plus difficile sur un écran d'ordinateur que sur papier. Cela requiert un temps de lecture des questions plus long et renforce le sentiment de manque de temps. Les étudiants n'ont pas la possibilité de mettre en place leurs stratégies habituelles comme le surlignage et les annotations, ce qui augmente leur distractibilité et le risque d'erreur. De plus, l'impossibilité de retour en arrière est aussi souvent évoquée. Cela perturbe à nouveau les stratégies habituelles comme commencer par les questions les plus complexes ou celles qui valent le plus de points, ou encore répondre à toutes les questions puis prévoir une relecture orthographique.

Sept étudiants rapportent une concentration difficile et un stress augmenté dans l'environnement (matériel ou connexion Internet) où se déroule l'évaluation. Sept étudiants font également part de problème de communication avec l'équipe enseignante. 


\section{Tableau 2}

Les difficultés rencontrées lors des examens présentés en ligne par les étudiants en lien avec la situation de handicap

\begin{tabular}{|l|c|}
\hline \multicolumn{1}{|c|}{ Difficultés } & $\mathrm{N}(67)$ \\
\hline $\begin{array}{l}\text { 1. Durée des examens, manque de temps, difficulté à gérer } \\
\text { le temps }\end{array}$ & 29 \\
\hline $\begin{array}{c}\text { 2. Modifier le format questionnaire } \\
\text { Difficulté lecture sur ordi, examen sur ordi }\end{array}$ & 21 \\
\hline $\begin{array}{c}\text { 4. Environnement } \\
\text { Concentration difficile, stress }\end{array}$ & 7 \\
\hline Manière d'interagir avec profs, assistants & 7 \\
\hline
\end{tabular}

La seconde question ouverte était posée comme suit : « En lien avec votre situation de handicap, qu'estce qui a été mis en place et qui vous a particulièrement aidé pour les examens en ligne? » (tableau 3).

\section{Tableau 3}

L'aide mise en place lors des examens présentés en ligne par les étudiants en lien avec la situation de handicap

\begin{tabular}{|l|c|}
\hline \multicolumn{1}{|c|}{ Aide } & $\mathrm{N}(66)$ \\
\hline $\begin{array}{l}\text { 1. Temps supplémentaire } \\
\text { Particulièrement important, car réduit lors des } \\
\text { examens en distanciel }\end{array}$ & 39 \\
\hline $\begin{array}{l}\text { 2. Modalité examens, caractéristiques } \\
\text { Proposition d'un oral }\end{array}$ & 9 \\
\hline $\begin{array}{l}\text { 3. Format questionnaire sur ordi } \\
\text { Format adapté, zoom possible, écriture plus } \\
\text { confortable }\end{array}$ & 6 \\
\hline
\end{tabular}

La question temporelle est à nouveau évoquée par 39 étudiants. Ils énoncent que le temps supplémentaire dont ils ont bénéficié a été salvateur par la durée réduite des examens en distanciel. Neuf étudiants disent avoir profité d'une modification des modalités d'évaluation (par exemple : la proposition de remplacer un examen écrit par un examen oral ou la possibilité de sélectionner la tranche horaire qui leur convient le mieux). Six étudiants rapportent, quant à eux, un meilleur confort lors des évaluations sur ordinateur. Par exemple, une meilleure adaptation du questionnaire, une possibilité de zoomer ou une écriture avec un clavier plus confortable. 
La troisième question ouverte s'intéressait aux pistes d'amélioration à explorer : «En lien avec votre situation de handicap, qu'est-ce qui pourrait vous aider pour aborder plus sereinement la prochaine session d'examens? ».

\section{Tableau 4}

L'aide en lien avec la situation de handicap qui pourrait être mise en place lors des examens présentés en ligne par les ESH

\begin{tabular}{|l|c|}
\hline \multicolumn{1}{|c|}{ Aide } & $\mathrm{N}(60)$ \\
\hline $\begin{array}{l}\text { 1. Améliorer la communication } \\
\text { Informations suffisamment tôt } \\
\text { Consignes EBS précises } \\
\text { Confirmation des aménagements et du local } \\
=>\text { apporte de la sérénité }\end{array}$ & 20 \\
\hline $\begin{array}{l}\text { 2. Modifier les modalités de l'examen } \\
\text { Favoriser le présentiel pour les EBS } \\
\text { Possibilité de modifier les modalités (examen }\end{array}$ & 17 \\
\hline $\begin{array}{l}\text { Possibilité de lire et répondre en format papier } \\
\text { Possibilité de voyager dans le questionnaire }\end{array}$ & \\
\hline 4. Augmenter la durée de l'examen \\
$\begin{array}{l}\text { Tiers-temps pas toujours suffisant, car réduction } \\
\text { générale de la durée des examens en ligne } \\
\text { (limiter la tricherie) }\end{array}$
\end{tabular}

À cette question (tableau 4), 20 étudiants répondent qu'ils souhaitent une amélioration de la communication et cela concerne particulièrement : le rappel des aménagements, et ce, suffisamment tôt, et la réception de consignes spécifiques précises. Ils rapportent que cela participe à leur sentiment de sérénité. On peut aussi observer que 17 étudiants sont favorables à une plus grande souplesse concernant les modalités d'évaluation, comme une proposition systématique d'examens en présentiel pour les ESH ou une possibilité de présenter un examen oral en remplacement d'un examen écrit. Neuf étudiants souhaitent pouvoir modifier le format du questionnaire : soit en format papier, soit sur le plan de la navigation libre. Enfin, quelques étudiants (7) rappellent qu'une augmentation de la durée des examens leur serait utile. 


\section{Analyse des résultats}

Tout d'abord, en croisant les difficultés rencontrées, les aides appréciées et souhaitées, nous pouvons mettre en évidence les points suivants :

1) Les étudiants rapportent avoir été mis en difficulté concernant la gestion du temps. Certains en ont manqué, d'autres s'en sont sortis, car ils ont pu bénéficier d'un tiers-temps supplémentaire. Cet aménagement est l'un des plus fréquemment recensés (Fournier, Hubert et Careau, 2020) mettant bien en évidence la question centrale de la gestion du temps pour les ESH. Les origines de ce manque de temps sont multiples et variées comme le manque d'habitude ou la complication engendrée par la rédaction d'une réponse sur l'ordinateur en temps réel. Ils se questionnent cependant sur la réduction de la durée des examens en distanciel afin d'éviter la tricherie. II nous apparaît toutefois qu'il est possible de limiter le plagiat comme la tricherie par d'autres méthodes comme une approche de promotion de l'intégrité. II s'agit, par exemple, de faire signer un code d'honneur aux étudiants les incitant à adopter un comportement éthique lors des évaluations ou de leur proposer une formation en ligne sur l'intégrité (Béland, Bureau et Peters, 2020).

2) Le format du questionnaire fait aussi partie des préoccupations des étudiants. Pour ceux présentant des troubles de l'apprentissage ou de l'attention, les examens sur ordinateur viennent perturber les stratégies mises en place depuis parfois plusieurs années. Par exemple, l'impossibilité de retour en arrière les empêche d'organiser leur manière de répondre aux questions d'examen. Une stratégie efficace étant de lire tous les items et de commencer à répondre aux plus difficiles ou à ceux rapportant le plus de points tant que leur attention est à son maximum. Une autre méthode, qui consiste à surligner les éléments importants sur la copie d'examen, est rendue impossible dans certains dispositifs sur ordinateur.

3) Les étudiants sont demandeurs de plus de souplesse sur le plan des modalités d'évaluation. En fonction des difficultés qu'ils présentent, pouvoir remplacer un examen oral par un examen écrit ou inversement, avoir la possibilité de présenter son évaluation écrite sur ordinateur sont des choix vécus comme très précieux. Nous remarquons cependant que les professeurs se montrent souvent très frileux à accepter ce type d'aménagements. Toucher aux modalités d'évaluation renvoie les professeurs à la question de l'égalité entre étudiants, qu'ils essayent de préserver. La gestion de l'équité entre les étudiants consiste à mettre en place des mesures pour atteindre l'égalité en rompant avec l'uniformité des modalités, car elles sont inéquitables pour les ESH. Un travail reste à faire auprès des enseignants afin de promouvoir l'équité.

4) La question de la communication apparaît centrale. II importe que les facultés veillent à communiquer les informations pertinentes relatives aux aménagements, et ce, le plus précocement possible. Cela participe au climat de sérénité bénéfique aux étudiants pour aborder leur session d'évaluations. Une communication de qualité diminue la charge mentale et le stress des étudiants qui se sentent davantage préparés (Detroz, Malay et Crahay, 2020).

Ensuite, nous constatons que les difficultés mises en évidence par les ESH (de manière exacerbée) le sont également dans une large mesure par les étudiants tout-venant. Cela doit participer à une réflexion plus globale. En effet, améliorer les conditions d'évaluation en se référant aux besoins des ESH permettrait d'assurer des conditions d'évaluation plus universelles. Il est également à noter (même si cela n'apparaît pas directement dans les résultats présentés ici) que quelques étudiants (avec troubles anxieux surtout) sont rassurés par les évaluations à distance et se montrent inquiets du retour en présentiel. Ils devront retrouver leurs marques dans un environnement plus insécurisant pour eux et auquel ils ont, de plus, perdu l'habitude de se confronter (Villeneuve et Dagneau, 2021). 
Nous observons que les ESH sont en attente de sérénité pour aborder leur session d'évaluation. Les aménagements dont ils bénéficient sont profitables en tant que tels, mais tout autant en permettant aux étudiants d'aborder leurs examens de manière posée, sans crainte d'être mis en difficulté par leur trouble. Il est important que le corps professoral en prenne conscience. Lors d'un séminaire interne à l'Université, les enseignants ont eu l'occasion de passer un test dans les conditions d'examens à distance. Cela leur a permis de prendre conscience du stress et des difficultés que cela pouvait engendrer. Detroz et al. (2020) remettent en question l'utilisation des outils antifraudes lors des évaluations tout en étant conscients que ces derniers engendrent de l'anxiété chez les étudiants.

II nous importe de souligner que la peur de la tricherie lors des examens en distanciel peut se comprendre et qu'elle engendre de nouvelles modalités d'évaluation. II s'agit cependant de rester prudents et de se questionner : sommes-nous assurés que les évaluations permettent bien de mesurer l'acquisition des concepts et qu'elles ne sont pas biaisées par le stress généré (par exemple: rapidité de réponse demandée)? La peur de la tricherie ne doit pas conditionner de manière disproportionnée la mise en place des aménagements. II s'agit, selon Detroz et al. (2020, p. 114), d'« équilibrer l'évaluation entre bienveillance et contrôle ». Cet équilibre n'est pas simple à atteindre entre " anxiété de l'étudiant liée à des outils de contrôle inhabituels et volonté de l'enseignant de préserver l'intégrité de son examen ».

\section{En guise de conclusions et de perspectives}

Nous considérons cette enquête comme une première étape dans le processus d'amélioration de l'enseignement inclusif. L'idéal serait d'aller un pas plus loin et de rencontrer les étudiants et les enseignants afin de creuser, d'analyser, et de décortiquer la manière dont les choses se sont passées pour chacun des acteurs. Cela permettrait d'avancer vers un système d'évaluation en équilibre entre les besoins des ESH et les exigences du cadre universitaire.

Quelques conseils peuvent aussi être communiqués aux ESH. Ils ne doivent pas hésiter à être proactifs, à communiquer leurs besoins (aménagements, informations, sérénité...). Ils se montrent parfois réticents, pensant que leur avis ne sera pas pris en compte, mais l'accès aux aménagements raisonnables est un droit qu'ils peuvent revendiquer en toute équité. L'interpellation peut être réalisée seule, mais elle aura davantage de poids en groupe ou via les délégués de cours. Le décret relatif à l'enseignement inclusif date de 2014 en Fédération Wallonie-Bruxelles. Il est par conséquent normal qu'il suscite encore beaucoup de questions et d'interpellations du côté du corps professoral.

Cette situation inédite de pandémie a demandé de nombreuses adaptations rapides. Elle a démontré, comme le soulignent Villeneuve et Dagneau (2021), " que l'exercice des droits des étudiants en situation de handicap n'est jamais acquis. Des événements imprévus peuvent entraîner l'apparition de nouveaux obstacles susceptibles de se transformer en situation discriminatoire » (p. 199). Mais elle a aussi été une opportunité pour d'intéressantes avancées. Elle a permis, par exemple, de dépasser les freins concernant l'utilisation de l'ordinateur lors des évaluations ou de l'enregistrement des cours. Souhaitons que ces avancées se poursuivent de manière raisonnée dans le temps. 


\section{Liste de références}

Audet, L. (2011). Les pratiques et défis de l'évaluation en ligne, REFAD. https://archives.refad.ca/evaluation en ligne.pdf

Béland, S., Bureau, J. S. et Peters, M. (2020). Plagier en temps de pandémie. Évaluer. Journal international de recherche en éducation et formation, numéro hors-série, 1, 35-40. https://journal.admee.org/index.php/ejiref/article/view/216

Biggs, J. (1996). Enhancing teaching through constructive alignment. Higher Education, 32, 347-364. http://doi.org/10.1007/BF00138871

Detroz, P., Malay, L. et Crahay, V. (2020). Une démarche structurée pour définir quelques conseils en vue de limiter l'impact de la pandémie sur l'évaluation de nos étudiants. Évaluer. Journal international de recherche en éducation et formation, numéro hors-série, 1, 97-110. https://journal.admee.org/index.php/ejiref/article/view/223

Detroz, P., Tessaro, W. et Younès, N. (2020). Pour la relance d'une évaluation congruente à l'université. Évaluer. Journal international de recherche en éducation et formation, numéro hors-série, 1, 111-119. https://journal.admee.org/index.php/ejiref/article/view/224

Fournier, A. L., Hubert, B. et Careau, L. (2020). Obstacles et facilitateurs perçus par les étudiants en situation de handicap à l'université et l'appréciation des services. Canadian Journal of Education, 43(2), 465-497. https://journals.sfu.ca/cje/index.php/cje-rce/article/view/4131

Keunen, V. (2020). Les aménagements raisonnables. Optimiser la réussite scolaire des élèves à besoins spécifiques. Erasme.

Liang, X. et Creasy, K. (2004). Classroom assessment in web-based instructional environment: Instructors' experience. Practical Assessment, Research, and Evaluation, 9(7). https://scholarworks.umass.edu/pare/vol9/iss1/7/

Nizet, I., Leroux, J. L, Deaudelin, C., Béland, S. et Goulet, J. (2016). Bilan de pratiques évaluatives des apprentissages à distance en contexte de formation universitaire. Revue internationale de pédagogie de l'enseignement supérieur, 32(2). http://journals.openedition.org/ripes/1073

Villeneuve, H. et Dagneau, B. (2021). Pandémie de COVID-19 et ses impacts pour les étudiants en situation de handicap : l'expérience vécue à l'Université Laval. Aequitas Revue de développement humain, handicap et changement social, 27(1), 193-200. 